\title{
Gas Assisted E-beam Lithography Using the JSM-7600F Ultra-High Resolution Schottky FEG-SEM and OmniGIS
}

\author{
Natasha Erdman*, Anthony Laudate* and Cheryl Hartfield** \\ * JEOL USA INC., 11 Dearborn Rd, Peabody, MA 01960 \\ ** Omniprobe, 10410 Miller Rd, Dallas, TX 75238
}

Electron beam-induced deposition (EBID) typically refers to a process of decomposing gaseous molecules by the electron beam in an SEM leading to deposition of non-volatile fragments onto a substrate (see Fig. 1a). The SEM beam provides high spatial resolution (close to $1 \mathrm{~nm}$ ) with the ability to produce free-standing, three-dimensional structures of metal or insulator based on the precursor that is used for EBID. The precursor material can be gas, liquid or solid. Liquid or solids are gasified prior to deposition, usually through vaporization or sublimation, and introduced into the high-vacuum chamber of the electron microscope at a precisely controlled rate; a solid precursor is sublimated by the electron beam itself. The deposited structure can then be immediately characterized either via imaging or analytical techniques without the need to transfer the specimen out of the SEM chamber.

Nanostructures of virtually any three-dimensional shape can be deposited using computercontrolled scanning of the electron beam. We utilized the NPGS (Nabity Pattern Generation System) in combination with the Omniprobe OmniGIS ${ }^{\mathrm{TM}}$ gas injection system for creating various structures using the JSM-7600F Schottky FEG-SEM. Fig. 1b shows the general setup of the system. The OmniGIS ${ }^{\mathrm{TM}}$ is a chemical precursor delivery system that can support up to three different crucibles carrying different precursors, as well as two additional inert dry gases that can be used either as a carrier gas or a purge gas. The OmniGIS ${ }^{\mathrm{TM}}$ system is mounted onto the SEM using a single port, thus not interfering with other potential accessories and detectors.

In our experiments, the OmniGIS ${ }^{\mathrm{TM}}$ system mounted onto the JSM-7600F was equipped with three different precursors for deposition: $\mathrm{MeCpPtMe}_{3}(\mathrm{Pt}), \mathrm{W}(\mathrm{CO})_{6}(\mathrm{~W})$ and TEOS $\left(\mathrm{SiO}_{2}\right)$. We have performed a variety of experiments using NPGS patterns of wheels, lines, dots and circles. In particular, we focused of the variation of gas flow parameters for different precursors as well as electron beam parameters $(\mathrm{kV}$, spot size, WD) to obtain either the fastest deposition and/or smallest feature size. We will present results of our experiments showing the different patterns as well as some of the smaller feature sizes attainable on bulk specimens (Fig. 2). We will also show the overall compositional analysis of the deposits as well as some electrical measurement of resistivity of the individual patterns. We will discuss and compare the results of our experiments to other deposition techniques using either a dedicated e-beam tool, using a resist and subsequent liftout procedure, or ion-beam assisted deposition in FIB instruments.

\section{References:}

1. R.M. Langford, T.X. Wang, D. Ozkaya, Microelectronic Engineering 84 (2007) 784-788

2. S. Randolph, Critical Reviews of Solid State and Materials Sciences 31 (2006) 55. 


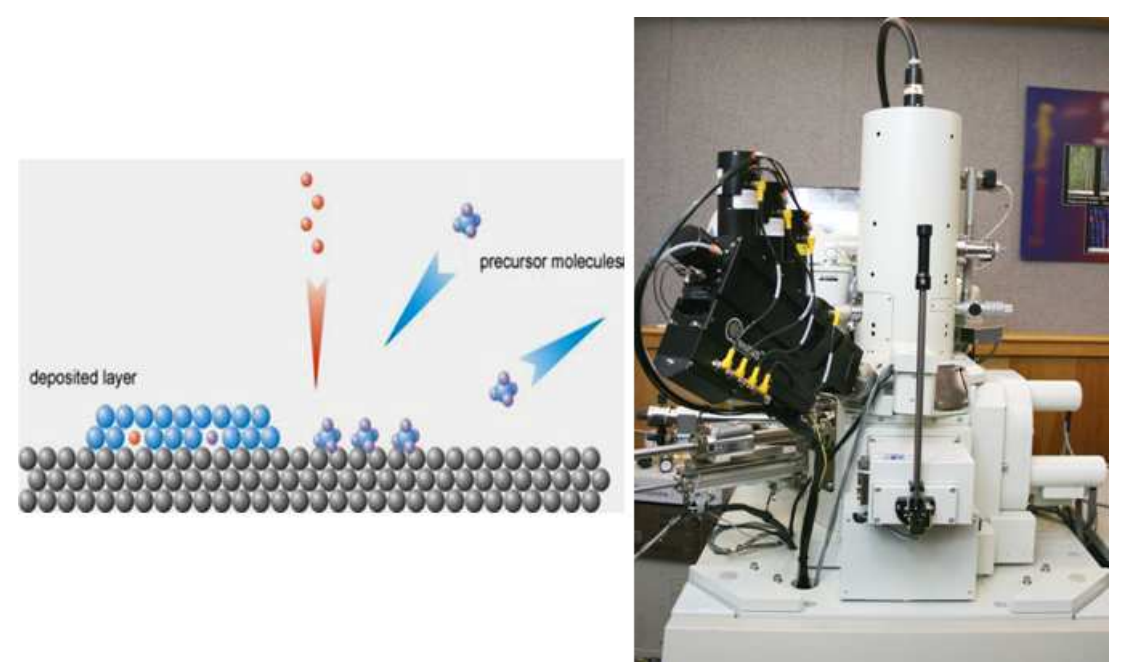

Fig.1. Left - general mechanism of gas assisted e-beam deposition. Right - setup of OmniGIS on JEOL JSM 7600F.

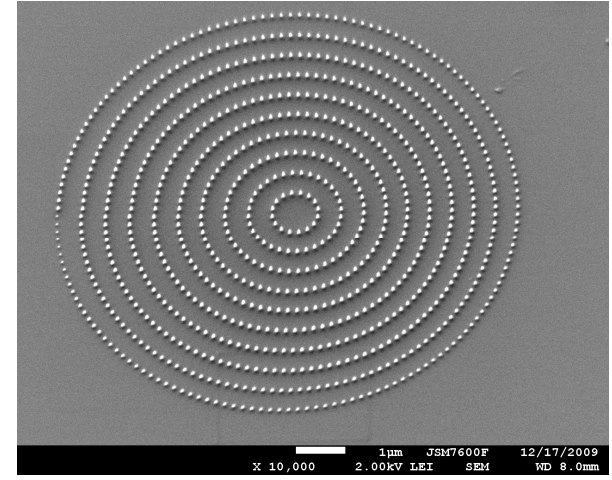

Circles, Pt deposition, $30 \mathrm{kV}, 1 \mathrm{nA}(7 \mathrm{~min})$

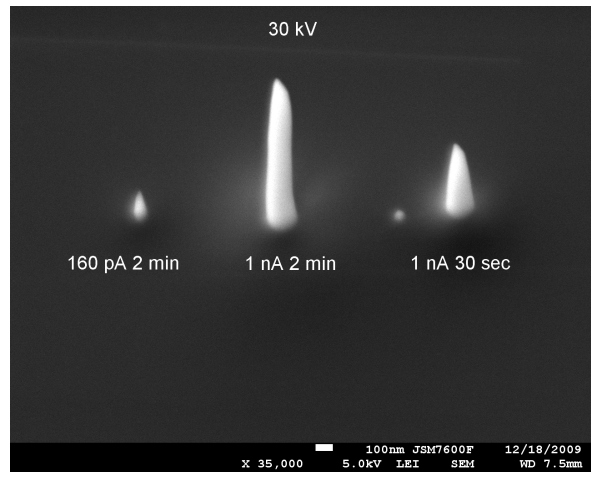

Pt pillars, grown in spot mode.

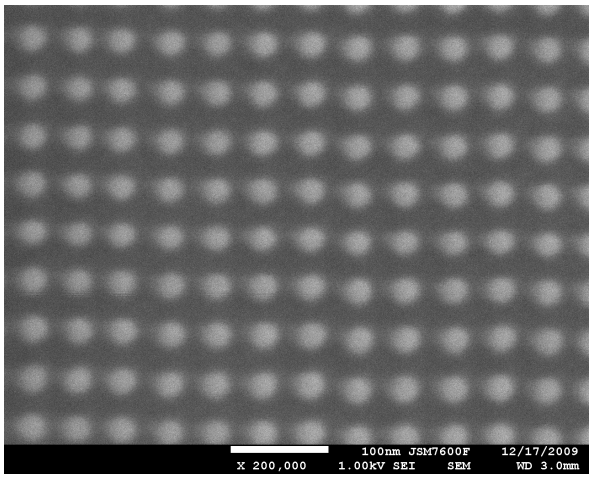

Pt deposition, $30 \mathrm{kV}, 1 \mathrm{nA}, 2.6 \mathrm{~min}$ $23 \mathrm{~nm}$ dots, $50 \mathrm{~nm}$ spacing

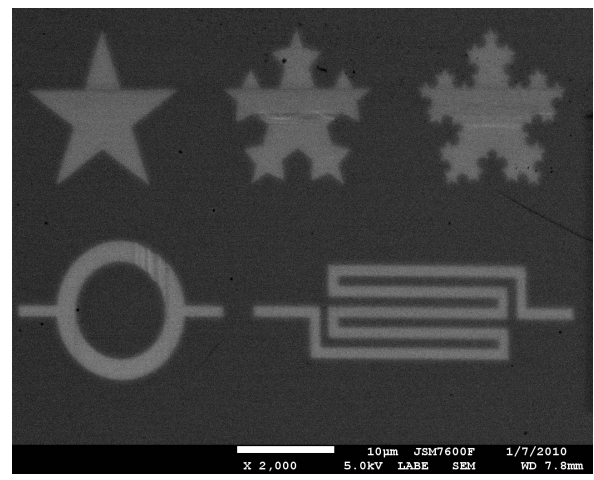

NPGS star pattern

Fig.2. Some results of the e-beam assisted depositions performed with OmniGIS on JEOL JSM$7600 F$ with NPGS system. 